\title{
Malpositioning of a nasogastric tube: a pitfall in the emergency department
}

\author{
Wei-Jing Lee • Reng-Hong Wu • Yi-Shien Chen • \\ Hung-Jung Lin
}

Received: 15 April 2008 / Accepted: 12 May 2008 / Published online: 17 June 2008

(C) Springer-Verlag London Ltd 2008

A 57-year-old man had a past history of diabetes mellitus controlled by oral hypoglycaemic agents. He was brought into the emergency department one day by paramedics after ingesting over $200 \mathrm{ml}$ of synthetic pyrethroid insecticide (alpha-cypermethrin). The patient was vomiting and agitated; therefore, a nasogastric tube was immediately inserted in order to perform gastric lavage. Routine chest X-ray was also done before admission (Fig. 1).

Placement of a nasogastric tube is indicated to decompress the stomach by aspiration of gastric contents, to introduce fluids and to assist in the clinical diagnosis of the ingested contents or gastric contents. Accidental misplacement of a nasogastric tube can occur in high-risk patients such as sedated patients, patients with weak cough reflex, endotracheally intubated patients and agitated patients [1]. Various methods to verify nasogastric tube placement in the stomach include the use of chest X-ray, aspiration of gastric content with the irrigating syringe, audible sounds of air entering the stomach while a $30 \mathrm{cc}$ air bolus is injected with the syringe, and able to talk without coughing, choking or cyanosis. It is important to verify nasogastric tube place-

W.-J. Lee $\cdot$ Y.-S. Chen $(\varangle) \cdot$ H.-J. Lin

Department of Emergency Medicine, Chi-Mei Medical Center, 901 Chung-Hua Road,

Yung-Kang City, Tainan 710, Taiwan

e-mail: saab931103@yahoo.com.tw

\section{R.-H. Wu}

Department of Radiology, Chi-Mei Medical Center,

901 Chung-Hua Road,

Yung-Kang City, Tainan 710, Taiwan

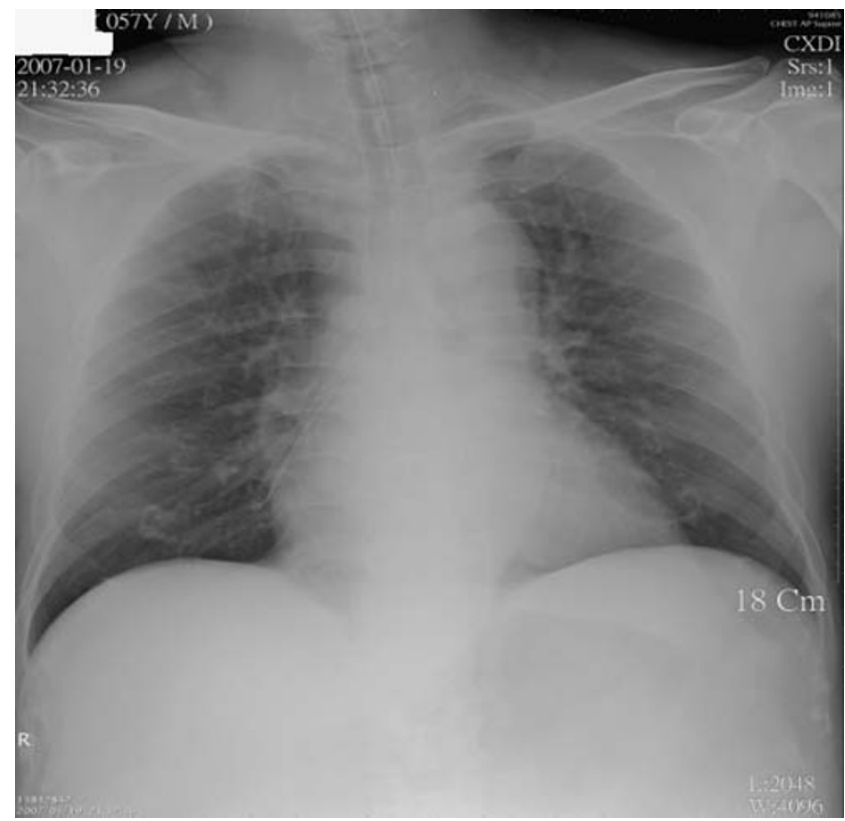

Fig. 1 Chest X-ray revealed nasogastric tube inserted into right main stem bronchus

ment in the stomach because the result of misplacement can be fatal such as aspiration pneumonitis or aspiration pneumonia.

\section{Reference}

1. Kawati R, Rubertsson S (2005) Malpositioning of fine bore feeding tube: a serious complication. Acta Anaesthesiol Scand 49(1):58-61 